Bio - grafía. Escritos sobre la Biología y su Enseñanza. ISSN 2027-1034

Edición Extraordinaria. p.p. 1204 - 1210

Memorias del IX Encuentro Nacional de Experiencias en Enseñanza de la Biología y

la Educación Ambiental. IV Congreso Nacional de Investigación en Enseñanza de la Biología.

\title{
TECNOLOGÍAS MÓVILES APLICADAS AL APRENDIZAJE DE LABOTÁNICA. PROYECTO QUICK NATURA
}

\author{
MOBILE TECHNOLOGIES APPLIED TO BOT ANICAL LEARNING. QUICK NATURA \\ PROJECT.
}

\author{
Ripoll Gómez, Sergio \\ Mayoral García-Berlanga, Olga \\ Azkárraga Testor, Jose Maria ${ }^{1}$
}

\section{RESUMEN:}

El proyecto Quick Natura consiste en acercar a las personas el entorno vegetal que les rodea de una manera sencilla y amena. Para ello, mediante las nuevas tecnologías y los códigos QR (Quick Response code), se conecta el mundo físico con el virtual en diferentes especies vegetales de la ciudad de Valencia (España). Cualquier usuario puede escanear estos códigos, situados de manera no invasiva en cada planta seleccionada, con un dispositivo móvil para acceder a una ficha virtual con información de fácil comprensión sobre la misma, así como a cuestionarios y la ubicación de otros códigos QR de la zona. El proyecto permite un uso tanto particular como tutelado, con un guía acompañante o docente, que pueda complementar la información obtenida a través de Quick Natura. Adicionalmente, se plantea la creación de una red de centros educativos que participen en el uso, así como en el desarrollo del proyecto, aportando nuevas fichas virtuales sobre especies vegetales a la base de datos pública del proyecto.

PALABRAS CLAVE: botánica, códigos $Q R$, didáctica, red de centros educativos, TIC

\section{ABSTRACT:}

The Quick Natura project intends to approach people to the environment related to plants around them in a simple and pleasant way by using the new technologies and QR codes (Quick Response codes), associating the physical world with the virtual world for different plant species of the city of Valencia. Any user can scan these codes in each selected plant, with a mobile device enabling access to easily understandable information about it in a virtual data sheet. Access is possible as well to questionnaires and locations of other QR codes in the area. The project is designed to allow its use both individually or mentored, with a guide that can supplement the information obtained through Quick Natura. Additionally, we contemplate the creation of a network of educational centres that participate in the use, as well as in the development of the project, contributing with new virtual plant species information to the shared database of the project.

\footnotetext{
${ }^{1}$ Departamento de Didáctica de las Ciencias Experimentales y Sociales. Universidad de Valencia, Facultad de Magisterio, Valencia. sergioripollgomez@gmail.com, olga.mayoral@uv.es, jose.m.azkarraga@uv.es
} 
Bio - grafía. Escritos sobre la Biología y su Enseñanza. ISSN 2027-1034

Edición Extraordinaria. p.p. 1204 - 1210

Memorias del IX Encuentro Nacional de Experiencias en Enseñanza de la Biología y

la Educación Ambiental. IV Congreso Nacional de Investigación en Enseñanza de la Biología.

KEYWORDS: botany, QR codes, didactics, network of educational centres, ICT

\section{INTRODUCCIÓN}

El presente proyecto surge de la necesidad de abordar tres de las principales dificultades que venimos encontrando en el aprendizaje y la enseñanza de la botánica en las aulas de los centros de Educación Secundaria en España.

El primero aspecto dificultoso, etiquetado como "ceguera hacia las plantas" (plant blindness) es un fenómeno que hace referencia a las dificultades que encuentran algunas personas para comprender los organismos vegetales, haciendo que pasen desapercibidos e incluso impidiendo que se perciba la importancia que tienen en nuestras vidas y en el medio natural (Bermúdez, 2015). Adicionalmente, encontramos en muchos libros de texto unos temarios reducidos, así como escasos experimentos propuestos, cuando hacen referencia al contexto vegetal en contrapartida a los dedicados al mundo animal (Balding, 2016).

El segundo factor está relacionado con las salidas al entorno. Este tipo de actividades resultan muy motivadoras y enriquecedoras, sirviendo en el contexto botánico, como refuerzo para interactuar directamente con el mundo vegetal. Sin embargo, requieren una cierta preparación previa, puesto que plantean diferentes barreras tales como: un coste económico añadido, seguro escolar, coordinación con otros profesores para organizar el calendario escolar, la elección de una localización concreta y la época del año adecuada para contextualizar la salida con el objetivo a lograr. Ciertos elementos vegetales no están presentes durante todo el año en las plantas y no todas las plantas presentan estos elementos de la misma manera y al mismo tiempo. Muchos docentes, según los centros donde imparten sus clases, no pueden afrontar estas dificultades (Graham, 2015).

El tercer aspecto a trabajar se halla en relación al uso de las nuevas tecnologías en las aulas. El avance de la tecnología implica a su vez un avance social que se manifiesta en nuestro modo de vida y en las aulas. Por ello, hoy en día es muy sencillo acceder a un ordenador o dispositivo móvil conectado a una red de datos y también encontrar alumnos de educación secundaria haciendo uso de ellos. El uso de estas tecnologías se invierte en diferentes actividades como la comunicación, la búsqueda de información y, en mayor medida, para el ocio (Herrera, 2014). Si bien las Tecnologías de la Información y la Comunicación (TIC) pueden resultar elementos de distracción en las aulas, el proyecto Quick Natura propone al docente incorporar experiencias didácticas y actitudes responsables con las nuevas tecnologías que de manera transversal ayuden a comprender la importancia de los diferentes usos que tienen los recursos tecnológicos.

Con el fin de ayudar a superar estos obstáculos, contribuyendo al mismo tiempo a las prácticas responsables en la educación ambiental, combinadas entre alumnos de diferentes centros educativos, el proyecto Quick Natura (Imagen 1) se plantea como una herramienta didáctica para reducir las barreras antes mencionadas. Mediante el uso de las nuevas tecnologías y a través de los dispositivos móviles, se recibe un conocimiento disponible tanto para el alumnado, profesorado o como para el visitante ocasional, que acerca el mundo vegetal de una manera sencilla, amena y rápida. 
Bio - grafía. Escritos sobre la Biología y su Enseñanza. ISSN 2027-1034

Edición Extraordinaria. p.p. 1204 - 1210

Memorias del IX Encuentro Nacional de Experiencias en Enseñanza de la Biología y la Educación Ambiental. IV Congreso Nacional de Investigación en Enseñanza de la Biología.

Quick Natura genera a su vez, un vínculo entre diferentes centros educativos y sus respectivas zonas naturales urbanas o periurbanas, a modo de red de intercambio de conocimiento donde los alumnos participan activamente en el desarrollo de los mismos.

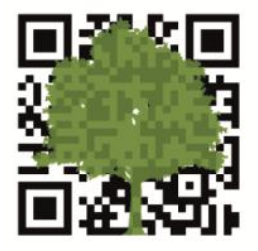

Imagen 1. Logotipo del proyecto Quick Natura

\section{METODOLOGÍA}

En el 2016 se seleccionaron 19 especies vegetales diferentes ubicadas en el recinto de la Facultat de Magisteri de la Universitat de València (España). De cada una de estas plantas se creó una ficha virtual alojada en los servidores de la Universitat de València a modo de página web (www.uv.es/quicknatura), donde se muestra información sobre ella. Esta información tiene un enfoque inicialmente anecdótico, para ir avanzado hacia una información más compleja, citando desde datos históricos y culturales hasta otros más técnicos y científicos (Imagen 2). De esta manera se generaron diferentes niveles de profundidad de conocimientos, donde el usuario puede elegir hasta qué punto desea llegar. Adicionalmente, todo término botánico utilizado aparece explicado en un glosario específico disponible en todo momento y las fotografías que acompañan a cada ficha muestran detalles de elementos vegetales que pueden no estar presentes durante todo el año.
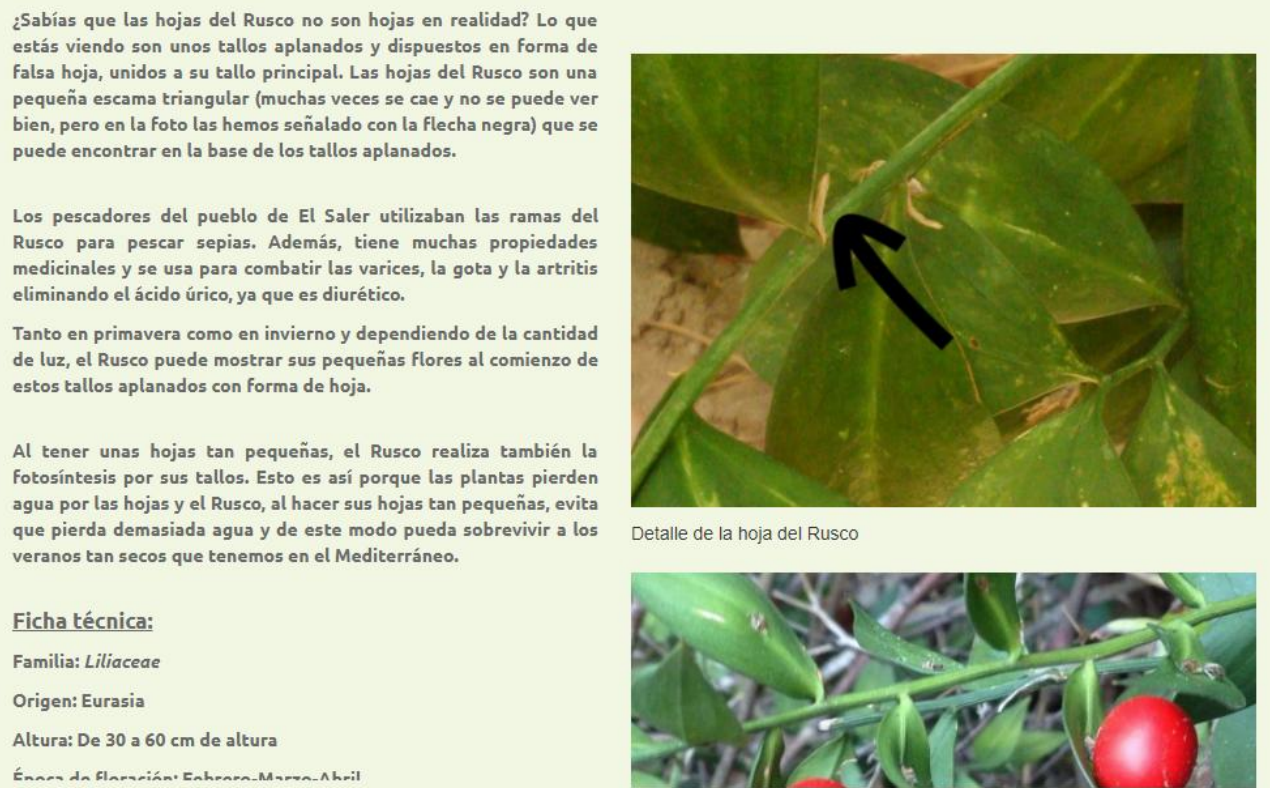

Detalle de la hoja del Rusco

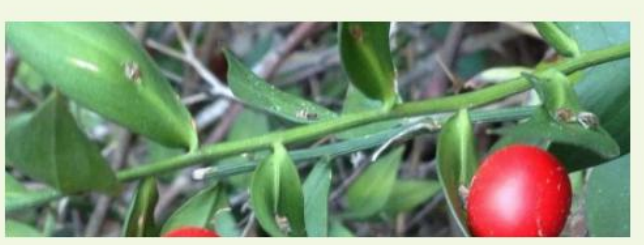

Imagen 2. Ejemplo de ficha virtual de una especie vegetal. Ruscus aculeatus 
Bio - grafía. Escritos sobre la Biología y su Enseñanza. ISSN 2027-1034

Edición Extraordinaria. p.p. 1204 - 1210

Memorias del IX Encuentro Nacional de Experiencias en Enseñanza de la Biología y la Educación Ambiental. IV Congreso Nacional de Investigación en Enseñanza de la Biología.

La ficha virtual de cada planta se asocia a un código QR generado por un servicio web. Este código QR se imprime y se pasa a colocar de una manera no invasiva en el espécimen concreto para acabar de conectar la ficha virtual con el mundo físico (Imagen 3). Para indicar al usuario que el código QR está haciendo referencia a datos botánicos se utilizó el logotipo del proyecto a modo de símbolo indicativo. Los usuarios de los códigos QR necesitan un dispositivo móvil conectado a una red de datos con una aplicación instalada que permita la lectura de códigos QR. Una vez escaneado el código de una planta, este redirige inmediatamente al usuario a la ficha creada en la página web.

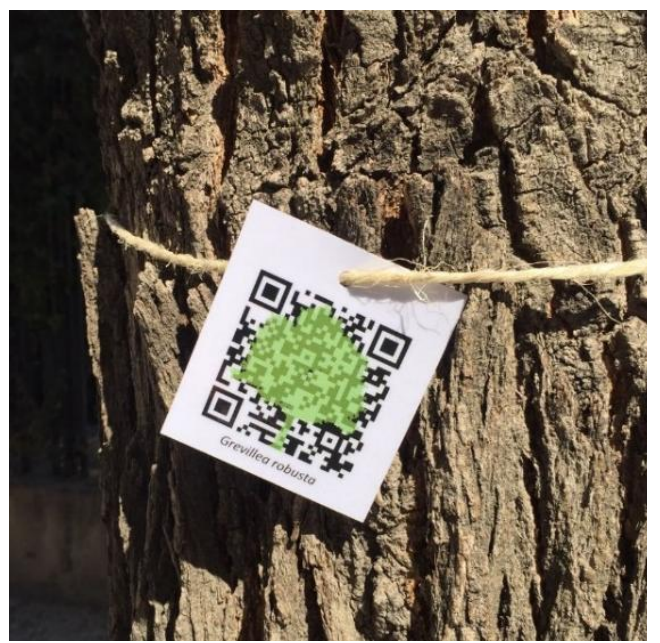

Imagen 3. Código QR del proyecto rodeando el tronco de Grevillea robusta

A su vez, cada ficha virtual posee un cuestionario con diferentes preguntas de tipo lógico-deductivo, según el nivel educativo del usuario, sobre la planta en la que se encuentra. Antes de acceder al cuestionario se ha de seleccionar el nivel educativo, para mostrar las preguntas acordes a dicho nivel. Al contestar las cuestiones planteadas, se recibe al instante información sobre la respuesta. Dichos cuestionarios fueron creados mediante la aplicación Hot Potatoes, siendo por ejemplo: “¿Cómo diferenciarías la Jacaranda de la Albizia si no tienen presentes flores ni frutos? Respuesta: Fijándose en la nerviación de las hojas" "¿A qué se debe que existan olivas negras y verdes en la misma especie (Olea europaea)? Respuesta: El color dependerá de la maduración del fruto.

Para crear itinerarios botánicos personalizados, se geolocalizó cada código QR mediante la aplicación de Google: My maps. Esta aplicación permite al usuario del código QR ver los otros códigos QR cercanos de Quick Natura, pudiendo elegir si continuar el recorrido, visitar simplemente los códigos de su interés, o guardar el mapa de Quick Natura para visualizarlo en otro momento (Imagen 4). La página web del proyecto se diseñó de manera que pueda ser visualizada en cualquier dispositivo móvil, smartphone, tablet, etc. (Imagen 5), no siendo necesario el uso de varios de estos dispositivos por grupo de usuarios. 
Bio - grafía. Escritos sobre la Biología y su Enseñanza. ISSN 2027-1034

Edición Extraordinaria. p.p. $1204-1210$

Memorias del IX Encuentro Nacional de Experiencias en Enseñanza de la Biología y la Educación Ambiental. IV Congreso Nacional de Investigación en Enseñanza de la Biología.

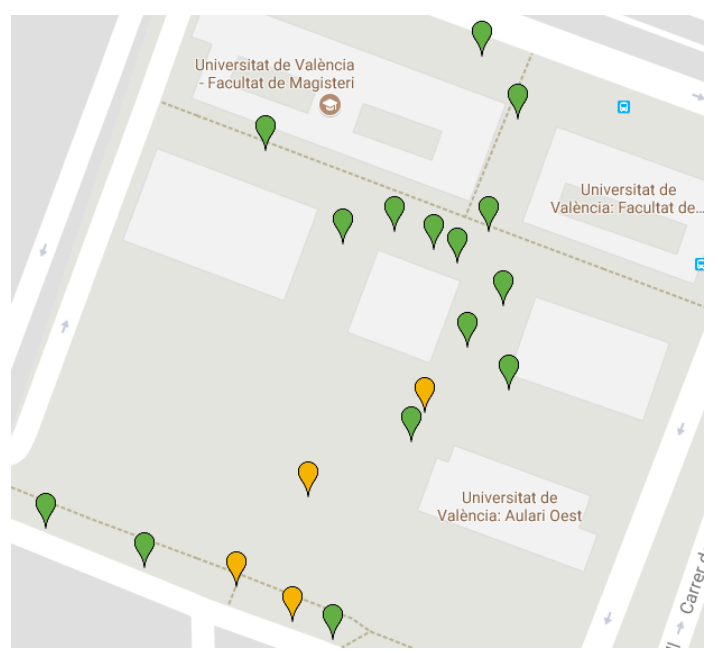

Imagen 4. Localización de los códigos QR en la Facultat de Magisteri de la Universitat de València mediante My maps

El proyecto permite suministrar los códigos QR en formato digital a los centros educativos interesados, pero también la posibilidad de que los propios alumnos creen el contenido de las fichas virtuales. El docente puede crear estos contenidos conjuntamente con sus alumnos y una vez revisados, enviarlos al equipo de Quick Natura para su posterior inserción en el servidor web. De esta manera los centros educativos pueden disponer del método y del espacio para sus propios códigos QR, personalizados según su zona o según los especímenes vegetales que deseen estudiar. Este último método sirve a su vez, para generar una red de conocimiento entre centros puesto que la base de datos de especímenes vegetales de Quick Natura está a disposición pública.

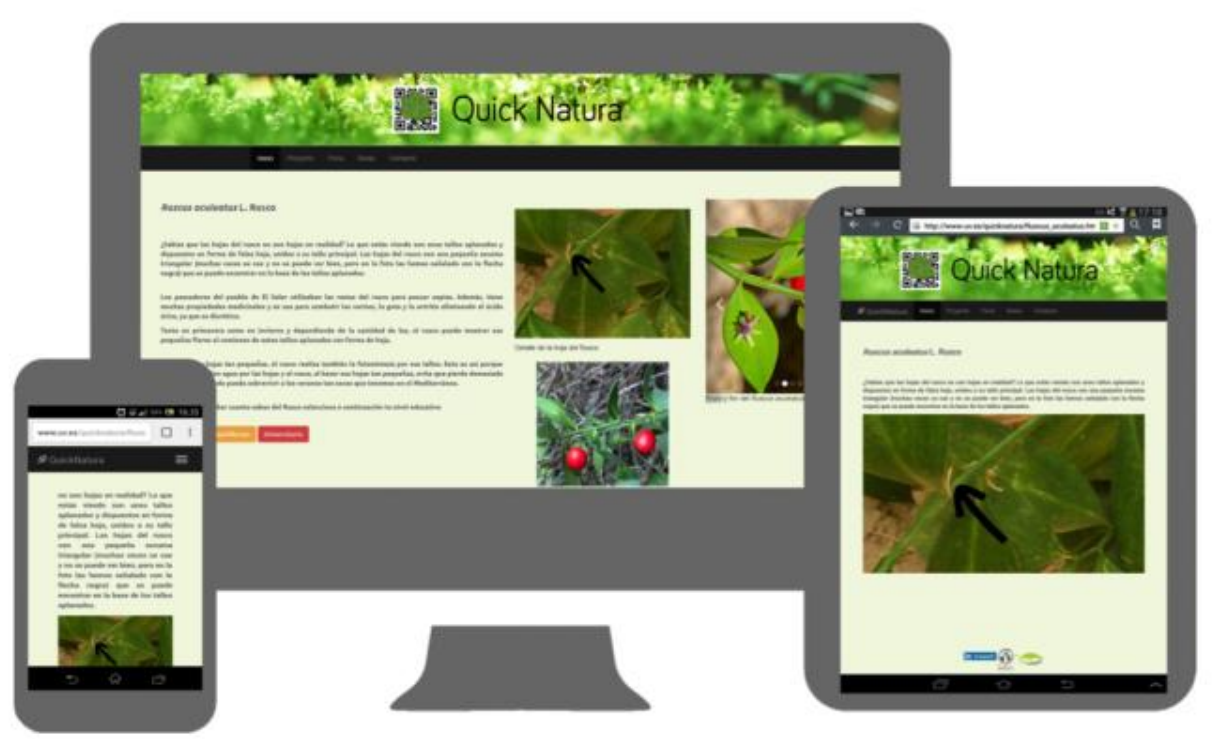


Bio - grafía. Escritos sobre la Biología y su Enseñanza. ISSN 2027-1034

Edición Extraordinaria. p.p. 1204 - 1210

Memorias del IX Encuentro Nacional de Experiencias en Enseñanza de la Biología y la Educación Ambiental. IV Congreso Nacional de Investigación en Enseñanza de la Biología.

Imagen 5. Ejemplo de visualización de la página web uww.uv.es/quicknatura en diferentes dispositivos

\section{RESULTADOS}

La página web alojada en el servidor de la Universitat de València utiliza la aplicación de Google: Google Analytics, mediante la cual registramos las visitas a la página web así como los códigos QR más visitados. El proyecto se inició con una prueba piloto. Durante la primera y segunda semana de su colocación en cada planta, se registró una media de 31 visitas a cada código. Teniendo en cuenta que no se realizó ningún tipo de publicidad para dar visibilidad al proyecto, consideramos que el proyecto resulta interesante tanto para transeúntes o visitantes, poniendo de manifiesto su potencialidad en otras zonas verdes urbanas o periurbanas, así como entornos de centros educativos.

El proyecto se encuentra gestionando su implantación en diferentes espacios públicos y jardines de la ciudad de Valencia, de manera que el visitante que lo desee reciba más información botánica, sin resultar invasiva, durante su visita a estos espacios.

Varios institutos de la ciudad de Valencia han mostrado su interés en formar parte de la red del proyecto. El Instituto de Educación Secundaria (IES) Lluís Vives ya tiene colocados códigos QR en diferentes plantas de su entorno (Imagen 6) y otros centros educativos han recibido códigos QR de especies vegetales, con su ficha virtual ya creadas, presentes en su entorno para su futura colocación. Por otro lado, otros centros educativos han mostrado interés en contribuir al proyecto, generando su propia información sobre la planta, para alojarla después en Quick Natura.

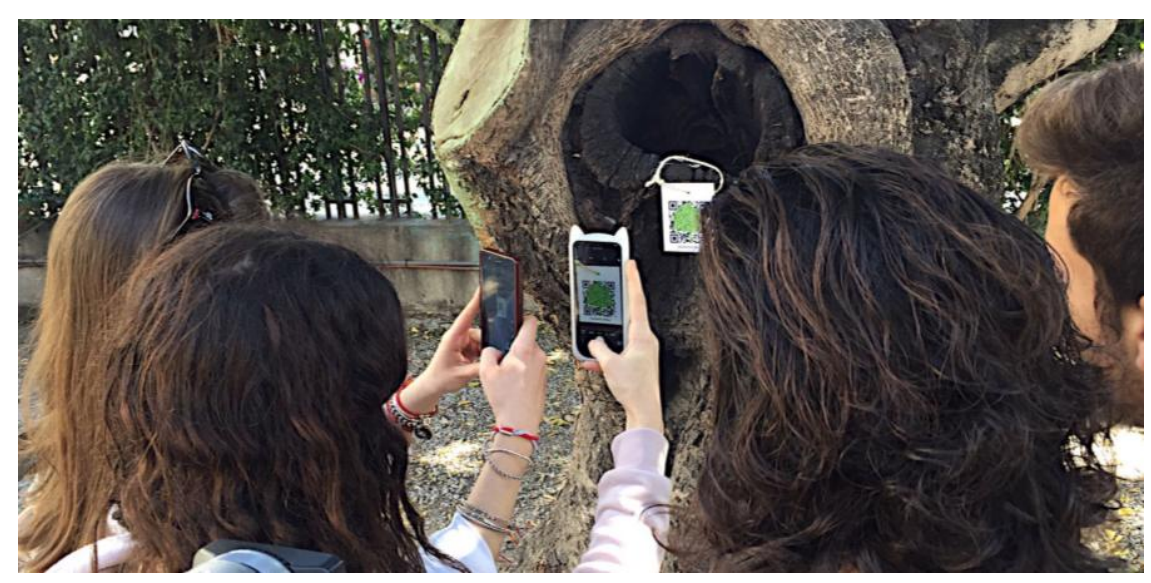

Imagen 6. Alumnos del Instituto de Educación Secundaria Lluís Vives haciendo uso de los códigos QR del proyecto 
Bio - grafía. Escritos sobre la Biología y su Enseñanza. ISSN 2027-1034

Edición Extraordinaria. p.p. 1204 - 1210

Memorias del IX Encuentro Nacional de Experiencias en Enseñanza de la Biología y

la Educación Ambiental. IV Congreso Nacional de Investigación en Enseñanza de la Biología.

\section{CONCLUSIONES}

El objetivo último del proyecto es, mediante la creación de una red de centros educativos y espacios verdes urbanos adheridos a la iniciativa conseguir una participación creciente por parte del alumnado en el proyecto Quick Natura, convirtiendo a los estudiantes en protagonistas de su aprendizaje. Ellos seleccionarán las diferentes especies a etiquetar, generar y filtrar la información a mostrar sobre ellas así como proponer preguntas a resolver de cada espécimen para el resto de usuarios. De esta manera, los alumnos de los propios centros estarán contribuyendo con información sobre la flora de su zona a la base de datos botánica del proyecto, a disposición del público general, pudiendo contrastar así la biodiversidad vegetal presente en diferentes zonas geográficas 0 incluso en diferentes espacios verdes urbanos de una misma ciudad. Complementariamente, se puede desarrollar una competencia cultural e histórica si los alumnos de un centro educativo establecen relaciones con la información botánica sobre otros espacios urbanos o periurbanos y no sólo del recinto del propio centro educativo. Este método permite, además de compartir información sobre diferentes especies vegetales, presentes o no en su localización, un aprendizaje personalizado donde cada usuario establece cuándo y hasta dónde aprender.

Se pretende, en definitiva, un acercamiento al mundo vegetal a través de la tecnología, cada vez más cercana en nuestra vida, para concienciar sobre la naturaleza que nos rodea y hacer de ella algo más cercano también.

\section{BIBLIOGRAFÍA}

Balding, M., y Williams, K.J.H. (2016). Plant blindness and the implications for plant conservation. Conservation Biology, 30: 1192-1199. doi:10.1111/cobi.12738

Bermúdez, J.M.M., y García Capocasa, C. (2015). Capítulo 11. La enseñanza de las plantas como un obstáculo educativo y los caracteres de visibilidad ecológica que pueden ayudar a superarlo: ¿qué especies consideran nativas los estudiantes de Córdoba? En G.M.A. Bermudez, y De Longhi, A.L. (Coordinadores), Retos para la enseñanza de la biodiversidad hoy. Aportes para la formación docente (pp. 293325). Córdoba: Universidad Nacional de Córdoba. 2015. ISBN: 978-987-707-003-3

Graham W.S., Boyd, M., Scott, L., y Colquhoun, D. (2015). Barriers To Biological Fieldwork: What Really Prevents Teaching Out of Doors? Journal of Biological Education, 49:2, 165-178, DOI: 10.1080/00219266.2014.914556

Herrera Sánchez, B., Aquiela Diez Irizar, G., y Buenabad Arias, M. A. (2014). El uso de los teléfonos móviles, las aplicaciones y su rendimiento académicos en los alumnos de la DES DACl. Revista lberoamericana para la Investigación y el Desarrollo Educativo, 12. ISSN $2007-7467$ 